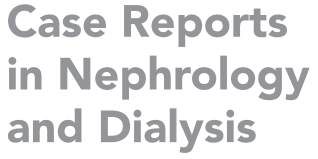

Case Reports and Dialysis

\title{
IgA Nephropathy Secondary to Ipilimumab Use
}

\author{
Sean C. Dougherty ${ }^{a}$ Nisa Desai ${ }^{a} \quad$ Helen P. Cathro ${ }^{b}$ Amanda Renaghan $^{a}$ \\ aDepartment of Internal Medicine, University of Virginia, Charlottesville, VA, USA; \\ ${ }^{b}$ Department of Pathology, University of Virginia, Charlottesville, VA, USA
}

\section{Keywords}

Acute renal failure $\cdot$ Histopathology $\cdot \lg A$ nephropathy $\cdot$ Kidney biopsy $\cdot$ Proteinuria

\begin{abstract}
Ipilimumab is a human monoclonal antibody targeting cytotoxic T-lymphocyte-associated protein 4 approved for the treatment of non-small-cell lung cancer (NSCLC) and other malignancies. Despite a high prevalence of other immune-related adverse events (irAEs), checkpoint inhibitor (CPI)-related nephrotoxicity has been reported less frequently. In this clinical case report, we describe the evaluation of a 70-year-old female with stage IV NSCLC who presented with nephrotic range proteinuria 4 weeks after receiving her first cycle of ipilimumab. She underwent a renal biopsy and was found to have IgA nephropathy that was presumed to be secondary to ipilimumab use, given recent initiation of therapy and clinical history. Unfortunately, despite prompt initiation of corticosteroids, her acute kidney injury progressed and she required hemodialysis, later transitioning to hospice. To our knowledge, this is one of few reported cases of IgA nephropathy secondary to CPI use. With increasing use of CPIs, this case further emphasizes the need for continued surveillance for irAEs, which can occur at any point in a patient's treatment course.
\end{abstract}

(c) 2021 The Author(s).

Published by S. Karger AG, Basel

\section{Introduction}

Immunotherapy has revolutionized the management of cancer ever since checkpoint inhibitors (CPIs) were first approved in 2011 [1]. CPIs are monoclonal antibodies that block inhibitory signals such as cytotoxic T-lymphocyte-associated protein 4 or programmed cell death 1, in turn resulting in an antitumor effect via increased T-cell activation [2]. 
Ipilimumab is an anti-cytotoxic T-lymphocyte-associated protein 4 antibody used as monotherapy or in combination with other CPIs that have been approved for the treatment of a number of malignancies, including non-small-cell lung cancer (NSCLC) [3].

Despite the efficacy of CPIs, immune-related adverse events (irAEs) are well documented and numerous, with dermatitis, enterocolitis, hypophysitis, cytopenias, and autoimmune encephalitis reported most commonly [4-7]. These irAEs can occur at any point during a patient's treatment course, and the mainstays of therapy are discontinuation of the offending agent and corticosteroids. Despite the increased frequency with which CPI-induced dermatitis and enterocolitis occur, irAEs with renal involvement are rare. Acute tubulointerstitial nephritis is the most commonly identified pathology, with biopsy-proven IgA nephropathy being an exceedingly rare entity [8-10].

In this clinical case report, we detail the presentation, evaluation, diagnosis, and management of a case of nephrotic syndrome attributed to IgA nephropathy secondary to ipilimumab that was unfortunately recalcitrant to corticosteroids and eventually fatal.

\section{Case Presentation}

A 70-year-old female with a past medical history of stage IV NSCLC with metastatic disease to the bones and liver presented with lower extremity edema. She reported 2 weeks of swelling that initially began around her eyes and face that then progressed inferiorly to her abdomen and lower extremities. She had gained 12 pounds over a similar time frame and noted new, progressively worsening hematuria with frothy urine. She denied fever or recent illness, although she had endorse dyspnea with dry cough and orthopnea. She also had a new erythematous maculopapular rash on her face over her eyelids, cheeks, and nose. She had received her first cycle of ipilimumab for the treatment of her lung cancer 4 weeks prior.

She was diagnosed with NSCLC approximately 2 years prior and completed 6 cycles of carboplatin, pemetrexed, and pembrolizumab with good initial response to therapy, followed by maintenance therapy with pemetrexed and pembrolizumab and progression of disease. Her treatment course was complicated by primary adrenal insufficiency secondary to pembrolizumab use, for which she was initially treated with pulse steroids (1 year prior to current presentation) and remained on oral prednisone ( $5 \mathrm{mg}$ by mouth daily). She had not received pembrolizumab for 3 months prior to her current presentation. Her other medical history included hypertension, gastroesophageal reflux disease, and osteoporosis. She continued to smoke approximately 1 pack of cigarettes per day.

On physical examination, her temperature was $37.1^{\circ} \mathrm{C}$, blood pressure was $192 / 104 \mathrm{~mm}$ $\mathrm{Hg}$, heart rate was 73 beats per minute, respiratory rate was 20 breaths per minute, and oxygen saturation was $94 \%$ on room air. She appeared to be in no acute distress and did not have evidence of jugular venous distention, though did have faint rales at the bases of the lungs, bilaterally. Her cardiovascular exam was unremarkable. Her abdominal exam did not have a fluid wave. She had 1+ edema of the bilateral lower extremities and a malar-like rash across the face that did not spare the nasolabial folds. She was alert, oriented, and mentating appropriately.

A complete blood count revealed a white blood cell count of $12.28 \times 10^{3} / \mathrm{uL}$, hemoglobin of $11.7 \mathrm{~g} / \mathrm{dL}$, and platelets of $301 \times 10^{3} / \mathrm{uL}$. A complete metabolic panel revealed a potassium of $4.3 \mathrm{mmol} / \mathrm{L}$, bicarbonate of $25 \mathrm{mmol} / \mathrm{L}$, blood urea nitrogen of $29 \mathrm{mg} / \mathrm{dL}$, and creatinine of 2.5 $\mathrm{mg} / \mathrm{dL}$ (baseline $0.9 \mathrm{mg} / \mathrm{dL}$ prior to the initiation of ipilimumab - shown in Fig. 1). Hepatitis B and $C$ serologies, anti-glomerular basement membrane, myeloperoxidase, and proteinase- 3 antibodies were all negative. An antinuclear antibody was weakly positive at a titer of 1:160 in a speckled pattern. C3 and C4 complement levels were 163 and $46 \mathrm{mg} / \mathrm{dL}$, respectively.

\section{Karger'}




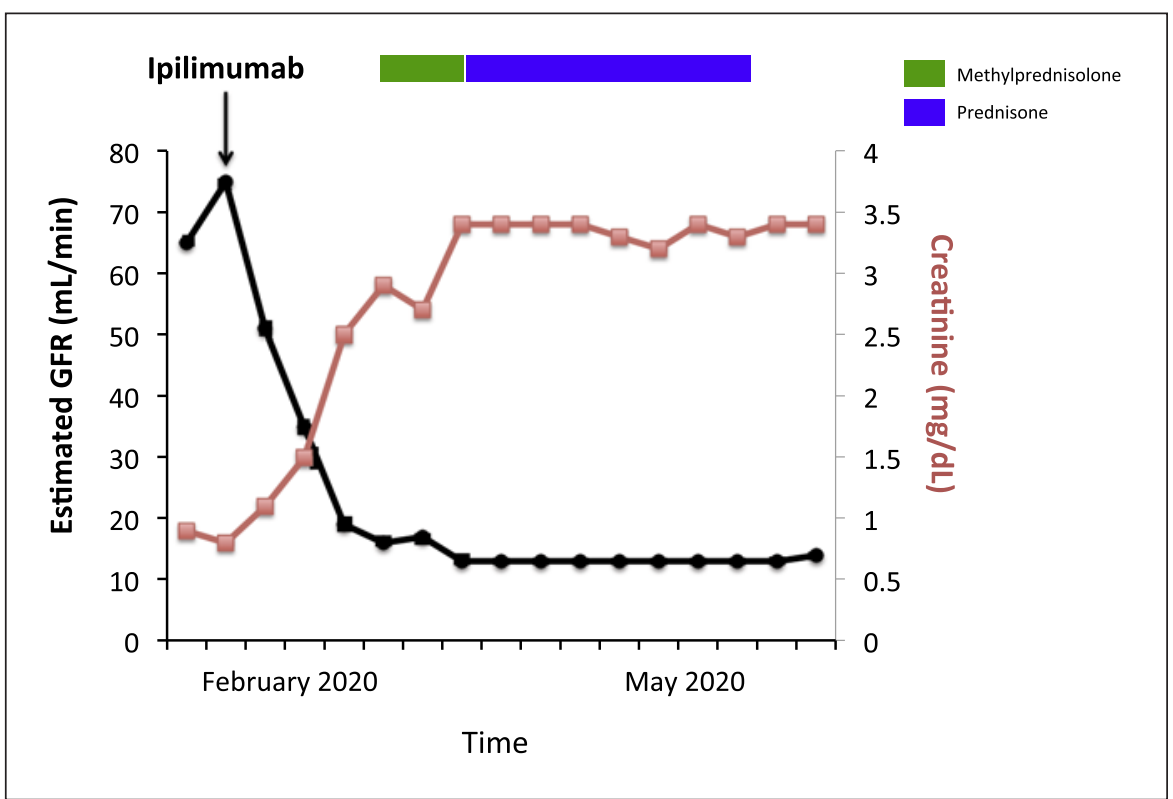

Fig. 1. Estimated glomerular filtration rate and creatinine plotted against time prior to and following initiation of ipilimumab (black arrow).

Serum and urine protein electrophoreses did not reveal evidence of an M-spike. Kappa-free light chains were $4.23 \mathrm{mg} / \mathrm{dL}$ and lambda-free light chains were $2.02 \mathrm{mg} / \mathrm{dL}$, yielding a kappa/lambda-free light chain ratio of 2.09. A urinalysis obtained was light-brown in color, had 3+ proteinuria, a moderate amount of blood, negative nitrites, small leukocyte esterase, $>50$ dysmorphic red blood cells per high powered field, 30-50 white blood cells per high powered field, and granular casts (see Table 1 for complete laboratory analysis). A chest x-ray showed interval development of small bilateral pleural effusions and multiple parenchymal opacities consistent with her known lung cancer. A renal ultrasound revealed normally appearing kidneys without evidence of renal vein thrombosis.

Due to concern for CPI-induced nephritis, she was started on methylprednisolone, $1 \mathrm{mg} / \mathrm{kg}$ IV twice daily on day 2 of hospitalization. She underwent a renal biopsy the following day; light microscopy revealed 39 glomeruli, one of which was globally sclerotic and 5 of which had cellular crescents. There was mesangial and endocapillary hypercellularity, the latter including neutrophils. Acute tubular damage, mild lymphocytic tubulitis with a focus of a few eosinophils, and fresh erythrocytic casts were also seen (shown in Fig. 2a, b). Immunofluorescence revealed mesangial staining with IgA (3+), C3 (1+), and kappa light chain (2+) (shown in Fig. 2d, e). There was no significant glomerular or tubular staining with IgG, IgM, C1q, or lamba light chain. Staining for IgG subunits showed no staining with IgG1, trace segmental staining with IgG2, and no staining with IgG3 or IgG4. Electron microscopy did not reveal evidence of immune complex-type electron dense deposits; however, analysis of the samples was limited by retroprocessing artifact. Overall, the biopsy results were thought to be most consistent with IgA nephropathy.

The patient was later discharged on a prolonged prednisone taper, receiving $60 \mathrm{mg}$ by mouth twice daily for 8 weeks. She did not receive further ipilimumab infusions. Unfortunately, her renal function continued to decline, and she was briefly placed on hemodialysis. Once hemodialysis was discontinued, she was subsequently readmitted for bilateral pleural effusions and worsening renal function, then elected not to pursue further dialysis and was discharged on hospice. 
Case Reports

in Nephrology and Dialysis

\begin{tabular}{l|l}
\hline Case Rep Nephrol Dial 2021;11:327-333 \\
\hline DOI: 10.1159/000519169 & $\begin{array}{l}\text { ○ 2021 The Author(s). Published by S. Karger AG, Basel } \\
\text { www.karger.com/cnd }\end{array}$ \\
\hline
\end{tabular}

Dougherty et al.: IgA Nephropathy Secondary to Ipilimumab Use

Table 1. Complete laboratory analysis

\begin{tabular}{lll}
\hline Test & Result & Reference range \\
\hline Complete blood count & & \\
White blood cells, $\times 10^{9} / \mathrm{L}$ & 12.28 & $4-11,000$ \\
Hemoglobin, g/dL & 11.7 & $12.0-16.0$ \\
Platelets, $\times 10^{3} / \mathrm{L}$ & 301 & $150-450$ \\
Complete metabolic panel & & \\
Sodium, mmol/L & 140 & $136-145$ \\
Potassium & 4.3 & $3.4-4.8$ \\
Chloride & 109 & $98-107$ \\
Bicarbonate & 25 & $23-31$ \\
Blood urea nitrogen, mg/dL & 29 & $10-20$ \\
Creatinine & 2.5 & $0.6-1.1$ \\
AST & 28 & $<35 \mathrm{U} / \mathrm{L}$ \\
ALT & 15 & $<55 \mathrm{U} / \mathrm{L}$ \\
Total bilirubin, mg/dL & 0.3 & Negative \\
Urinalysis & &
\end{tabular}

\section{Discussion}

CPI use for the treatment of multiple hematologic and solid-tumor malignancies has increased significantly in the last decade. Despite their efficacy, CPI-associated irAEs can occur at any point during a patient's treatment course and significantly affect any organ system. Nephrotoxicity from CPI is rare, and the exact pathophysiology remains unclear [11]. Suggested mechanisms include direct lymphocytic infiltration of the renal interstitium, immune complex-mediated injury, a lupus-like nephropathy, IgA nephropathy, microangiopathic hemolytic anemia, or cytokine release [12]. Acute tubulointerstitial nephritis and glomerular disease have been reported as causes of immune-related kidney injury, with acute tubulointerstitial nephritis being the most common pathologic finding on biopsy. A large case series studying renal toxicity from CPIs recently showed that clinically, patients can develop acute kidney injury without additional symptoms [13]. 


Case Reports
in Nephrology
and Dialysis
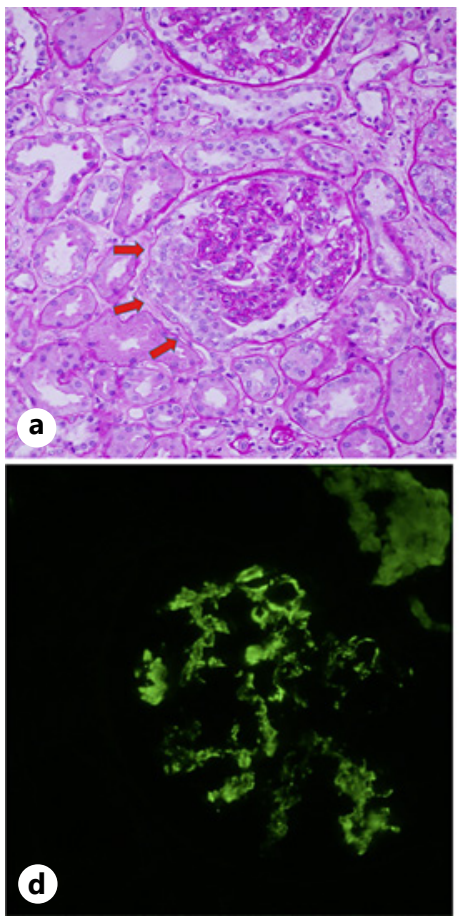

\begin{tabular}{l|l}
\hline Case Rep Nephrol Dial 2021;11:327-333 \\
\hline DOI: 10.1159/000519169 & $\begin{array}{l}\text { (c) 2021 The Author(s). Published by S. Karger AG, Basel } \\
\text { www.karger.com/cnd }\end{array}$ \\
\hline
\end{tabular}

Dougherty et al.: IgA Nephropathy Secondary to Ipilimumab Use

Fig. 2. a Central glomerulus displays a cellular crescent on the left; see red arrows (PAS. $\times 100)$. b A less damaged glomerulus shows severe mesangial expansion and mesangial hypercellularity (PAS. $\times 100$ ). c A few glomeruli demonstrated increased neutrophils within capillary lumens; see red arrows (Jones methenamine silver $\times 200)$. $\mathbf{d}$ Immunofluorescence for IgA stains the mesangium in a granular pattern $(3+$ on a scale of trace through $3+$ ). e Immunofluorescence for kappa light chain stains the mesangium in a granular pattern $(2+$ on a scale of trace through $3+$ ). $\mathbf{f}$ Areas of tubular atrophy were associated with interstitial fibrosis; see red arrows (Trichrome. $\times 40$ ).

Glomerular disease from CPIs has been described in prior reports. IgA nephropathy is the most common cause of primary glomerulonephritis; however, it has only rarely been reported previously in association with CPI use [9, 12]. Pathologic findings of acute kidney injury associated with CPI use are often indistinguishable from other drug-induced acute interstitial nephritides, typically revealing T-cell infiltration of the renal interstitium with occasional plasma cells and eosinophils. In primary IgA glomerulonephritis, patients typically present with hematuria that is often preceded by an upper respiratory infection. On biopsy, immunofluorescence shows mesangial dominant or co-dominant IgA deposits. Crescentic IgA nephropathy is defined as $>50 \%$ crescentic glomeruli on kidney biopsy and signals more aggressive disease. Nephrotic syndrome in IgA nephropathy is suggestive of advanced disease.

Given the disproportionate mesangial staining for kappa light chains on our patient's biopsy, an IgA-kappa monoclonal protein-associated nephropathy from a plasma cell dyscrasia was considered. This was thought to be less likely in the setting of normal serum and urine protein electrophoreses and a minimally elevated kappa/lambda-free light chain ratio. With endocapillary hypercellularity and neutrophils present, an IgA-dominant infection-associated glomerulonephritis was also considered; however, this was felt to be unlikely in the absence of preceding infectious symptoms or documented occult infection. The patient had no history of cirrhosis or other gastrointestinal disorder, respiratory tract disorder, or autoimmune disorder that would predispose to secondary IgA nephropathy. Further, while lung cancer itself can be associated with paraneoplastic (secondary) IgA nephropathy, her initial diagnosis was made 2 years prior to this current presentation. Given the temporal relation to the 
initiation of ipilimumab, the observed pathology, and clinical history, we believe that her acute kidney injury was an irAE related to ipilimumab.

The mainstays of treatment of irAEs include prompt initiation of corticosteroids and no further cycles of the causal agent. Tanabe et al. [14] recently reported a case of steroidresponsive IgA nephropathy secondary to nivolumab; however, like other irAEs, CPI-related nephrotoxicity may be recalcitrant to steroids in severe cases. For steroid-refractory cases, anti-TNF-alpha antibodies, mycophenylate mofetil, and calcineurin inhibitors may be effective [15]. While this patient's biopsy only had $13 \%$ crescents, the pathology was suggestive of aggressive disease, perhaps explaining her lack of response to corticosteroids. The fact that she was already on oral steroids at the time of diagnosis may have also affected her response to high-dose steroids in a negative manner. Further immunomodulating agents were not administered given the patient's decision to pursue hospice care. Overall, there are sparse case reports of CPI-associated IgA nephropathy. This case illustrates how such renal toxicity can lead to aggressive disease and a poor prognosis despite prompt initiation of high-dose corticosteroids.

\section{Statement of Ethics}

All procedures followed were in accordance with the ethical standards of the responsible committee on human experimentation and with the Helsinki Declaration of 1975, as revised in 2000. Written informed consent was obtained from the patient's legal next of kin as the patient was unfortunately deceased at the time of manuscript composition.

\section{Conflicts of Interest Statement}

The authors have no conflicts of interest to declare.

\section{Funding Sources}

This study did not receive any sponsorship or funding.

\section{Author Contributions}

S.C.D. aided in patient care and diagnosis of disease and wrote the manuscript under the mentorship of A.R.; N.D. aided in patient care and diagnosis of disease and wrote the manuscript under the mentorship of A.R.; H.P.C. aided in the diagnosis of disease, prepared histopathological images, and was the pathologist of record; A.R. supervised case report formulation, acquired and interpreted clinical data, and aided in the diagnosis of disease.

\section{References}

1 Gentzler R, Hall R, Kunk PR, Gaughan E, Dillon P, Slingluff CL Jr, et al. Beyond melanoma: inhibiting the PD-1/ PD-L1 pathway in solid tumors. Immunotherapy. 2016;8(5):583-600.

2 Sharma P, Allison JP. Immune checkpoint targeting in cancer therapy: toward combination strategies with curative potential. Cell. 2015;161(2):205-14.

3 Hellmann MD, Paz-Ares L, Bernabe Caro R, Zurawski B, Kim SW, Carcereny Costa E, et al. Nivolumab plus Ipilimumab in advanced non-small-cell lung cancer. N Engl J Med. 2019;381(21):2020-31. 
4 Postow MA, Sidlow R, Hellmann MD. Immune-related adverse events associated with immune checkpoint blockade. N Engl J Med. 2018;378(2):158-68.

5 Fecher LA, Agarwala SS, Hodi FS, Weber JS. Ipilimumab and its toxicities: a multidisciplinary approach. Oncologist. 2013;18(6):733-43.

6 Ayala FA, Dougherty SC, Swift W, Lapides DA. Overlapping NMDA-R and GFAP antibody autoimmune encephalitis after Nivolumab therapy. Neurol Clin Pract. 2020.

7 Dougherty SC, Lynch AC, Hall RD. Drug-induced immune-mediated thrombocytopenia secondary to durvalumab use. Clin Case Rep. 2021;9(6):e04227.

8 Izzedine H, Mateus C, Boutros C, Robert C, Rouvier P, Amoura Z, et al. Renal effects of immune checkpoint inhibitors. Nephrol Dial Transplant. 2017;32(6):936-42.

9 Kishi S, Minato M, Saijo A, Murakami N, Tamaki M, Matsuura M, et al. IgA nephropathy after nivolumab therapy for postoperative recurrence of lung squamous cell carcinoma. Intern Med. 2018;57(9):1259-63.

10 Oki R, Hirakawa Y, Kimura H, Honda K, Hinata M, Abe H, et al. Renal effects after pembrolizumab treatment for non-small cell lung carcinoma. Intern Med. 2020;59(7):977-81.

11 Wanchoo R, Karam S, Uppal NN, Barta VS, Deray G, Devoe C, et al. Adverse renal effects of immune checkpoint inhibitors: a narrative review. Am J Nephrol. 2017;45(2):160-9.

12 Mamlouk O, Selamet U, Machado S, Abdelrahim M, Glass WF, Tchakarov A, et al. Nephrotoxicity of immune checkpoint inhibitors beyond tubulointerstitial nephritis: single-center experience. J Immunother Cancer. 2019;7(1):2.

13 Cortazar FB, Marrone KA, Troxell ML, Ralto KM, Hoenig MP, Brahmer JR, et al. Clinicopathological features of acute kidney injury associated with immune checkpoint inhibitors. Kidney Int. 2016;90(3):638-47.

14 Tanabe K, Kanzaki H, Wada T, Nakashima Y, Sugiyama H, Okada H, et al. Nivolumab-induced IgA nephropathy in a patient with advanced gastric cancer: a case report. Medicine. 2020;99(21):e20464.

15 Brahmer JR, Lacchetti C, Schneider BJ, Atkins MB, Brassil KJ, Caterino JM, et al. Management of immune-related adverse events in patients treated with immune checkpoint inhibitor therapy: American society of clinical oncology clinical practice guideline. J Clin Oncol. 2018;36(17):1714-68. 